\title{
Global existence and exponential growth for a viscoelastic wave equation with dynamic boundary conditions
}

\author{
Stéphane Gerbi* and Belkacem Said-Houari ${ }^{\dagger}$
}

\begin{abstract}
The goal of this work is to study a model of the wave equation with dynamic boundary conditions and a viscoelastic term. First, applying the Faedo-Galerkin method combined with the fixed point theorem, we show the existence and uniqueness of a local in time solution. Second, we show that under some restrictions on the initial data, the solution continues to exist globally in time. On the other hand, if the interior source dominates the boundary damping, then the solution is unbounded and grows as an exponential function. In addition, in the absence of the strong damping, then the solution ceases to exist and blows up in finite time.
\end{abstract}

Keywords: Damped viscoelastic wave equations, global solutions, exponential growth, blow up in finite time, dynamic boundary conditions.

\section{Introduction}

We consider the following problem

$$
\begin{cases}u_{t t}-\Delta u-\alpha \Delta u_{t}+\int_{0}^{t} g(t-s) \Delta u(s) d s=|u|^{p-2} u, & x \in \Omega, t>0, \\ u(x, t)=0, & x \in \Gamma_{0}, t>0, \\ u_{t t}(x, t)=-\left[\frac{\partial u}{\partial \nu}(x, t)-\int_{0}^{t} g(t-s) \frac{\partial u}{\partial \nu}(x, s) d s+\frac{\alpha \partial u_{t}}{\partial \nu}(x, t)+h\left(u_{t}\right)\right] & x \in \Gamma_{1}, t>0, \\ u(x, 0)=u_{0}(x), \quad u_{t}(x, 0)=u_{1}(x) & x \in \Omega,\end{cases}
$$

where $u=u(x, t), t \geq 0, x \in \Omega, \Delta$ denotes the Laplacian operator with respect to the $x$ variable, $\Omega$ is a regular and bounded domain of $\mathbb{R}^{N},(N \geq 1), \partial \Omega=\Gamma_{0} \cup \Gamma_{1}$, mes $\left(\Gamma_{0}\right)>0, \Gamma_{0} \cap \Gamma_{1}=\varnothing$ and $\partial / \partial \nu$ denotes the unit outer normal derivative, $\alpha$ is a positive constant, $p>2, h$ and $g$ are functions whose properties will be discussed in the next section, $u_{0}, u_{1}$ are given functions.

Nowadays the wave equation with dynamic boundary conditions are used in a wide field of applications. See [24] for some applications. Problems similar to (1) arise (for example) in the modeling of longitudinal vibrations in a homogeneous bar in which there are viscous effects. The term $\Delta u_{t}$, indicates that the stress is proportional not only to the strain, but also to the strain rate, see [6] fore more details.

\footnotetext{
*Laboratoire de Mathématiques, Université de Savoie et CNRS, UMR-5128, 73376 Le Bourget du Lac, France, E-mail : stephane.gerbi@univ-savoie.fr,

${ }^{\dagger}$ Division of Mathematical and Computer Sciences and Engineering, King Abdullah University of Science and Technology (KAUST), Thuwal, KSA, E-mail: saidhouarib@yahoo.fr
} 
From the mathematical point of view, these problems do not neglect acceleration terms on the boundary. Such type of boundary conditions are usually called dynamic boundary conditions. They are not only important from the theoretical point of view but also arise in several physical applications. For instance in one space dimension and for $g=0$, problem (1) can modelize the dynamic evolution of a viscoelastic rod that is fixed at one end and has a tip mass attached to its free end. The dynamic boundary conditions represents Newton's law for the attached mass, (see $[5,2,8]$ for more details). In the two dimension space, as showed in [25] and in the references therein, these boundary conditions arise when we consider the transverse motion of a flexible membrane $\Omega$ whose boundary may be affected by the vibrations only in a region. Also some dynamic boundary conditions as in problem (1) appear when we assume that $\Omega$ is an exterior domain of $\mathbb{R}^{3}$ in which homogeneous fluid is at rest except for sound waves. Each point of the boundary is subjected to small normal displacements into the obstacle: this type of dynamic boundary conditions are known as acoustic boundary conditions, see [3] for more details.

Littman and Markus [19] considered a system which describe an elastic beam, linked at its free end to a rigid body. The whole system is governed by the Euler-Bernoulli Partial Differential Equations with dynamic boundary conditions. They used the classical semigroup methods to establish existence and uniqueness results while the asymptotic stabilization of the structure is achieved by the use of feedback boundary damping.

In [14] the author introduced the model

$$
u_{t t}-u_{x x}-u_{t x x}=0, \quad x \in(0, L), t>0,
$$

which describes the damped longitudinal vibrations of a homogeneous flexible horizontal rod of length $L$ when the end $x=0$ is rigidly fixed while the other end $x=L$ is free to move with an attached load. Thus she considered Dirichlet boundary condition at $x=0$ and dynamic boundary conditions at $x=L$, namely

$$
u_{t t}(L, t)=-\left[u_{x}+u_{t x}\right](L, t), \quad t>0 .
$$

By rewriting the whole system within the framework of the abstract theories of the so-called $B$ evolution theory, the existence of a unique solution in the strong sense has been shown. An exponential decay result was also proved in [15] for a problem related to (2)-(3), which describe the weakly damped vibrations of an extensible beam. See [15] for more details.

Subsequently, Zang and $\mathrm{Hu}$ [29], considered the problem

$$
u_{t t}-p\left(u_{x}\right)_{x t}-q\left(u_{x}\right)_{x}=0, \quad x \in(0,1), t>0
$$

with

$$
u(0, t)=0, \quad p\left(u_{x}\right)_{t}+q\left(u_{x}\right)(1, t)+k u_{t t}(1, t)=0, t \geq 0 .
$$

By using the Nakao inequality, and under appropriate conditions on $p$ and $q$, they established both exponential and polynomial decay rates for the energy depending on the form of the terms $p$ and $q$.

Recently, the present authors have considered, in [11] and [12], problem (1) with $g=0$ and a nonlinear boundary damping of the form $h\left(u_{t}\right)=\left|u_{t}\right|^{m-2} u_{t}$. A local existence result was obtained by combining the Faedo-Galerkin method with the contraction mapping theorem. Concerning the asymptotic behavior, the authors showed that the solution of such problem is unbounded and grows up exponentially when time goes to infinity provided that the initial data are large enough and 
the damping term is nonlinear. The blow up result was shown when the damping is linear (i.e. $m=2$ ). Also, we proved in [12] that under some restrictions on the exponents $m$ and $p$, we can always find initial data for which the solution is global in time and decays exponentially to zero. These results had been recently generalized for a wide range of nonlinearities in the equation and in the boundary term: the authors proved the local existence and uniqueness by a sophisticated application of the non linear semigroup theory, see [13].

In the absence of the strong damping $\alpha \Delta u_{t}$ and for Dirichlet boundary conditions on the whole boundary $\partial \Omega$, the question of blow up in finite time of problem (1) has been investigated by many authors. Messaoudi [23] showed that if the initial energy is negative and if the relaxation function $g$ satisfies the following assumption

$$
\int_{0}^{\infty} g(s) d s<\frac{(p / 2)-1}{(p / 2)-1+(1 / 2 p)},
$$

then the solutions blow up in finite time. In fact this last condition has been assumed by other researchers. See for instance [16, 17, 21, 22, 26, 28].

The main goal of this paper is to prove the local existence and to study the asymptotic behavior of the solution of problem (1).

One of the main questions is to show a blow-up result of the solution. This question is a difficult open problem, since in the presence of the strong damping term, i.e. when $\alpha \neq 0$, the problem has a parabolic structure, which means that the solution gains more regularity. However, in this paper, we give a partial answer to this question and show that for $\alpha \neq 0$ and for large initial data, the solution is unbounded and grows exponentially as $t$ goes to infinity. While for the case $\alpha=0$, the solution has been shown to blow up in finite time.

The main contribution of this paper in this blow up result is the following: the exponential growth and blow-up results hold without making the assumption (4). In fact the only requirement is that the exponent $p$ has to be large enough which is a condition much weaker than condition (4). Moreover, unlike in the works of Messaoudi and coworkers, we do not assume any polynomial structure on the damping term $h\left(u_{t}\right)$, to obtain an exponential growth of the solution or a blow up in finite time.

This paper is organized as follows: firstly, applying the Faedo-Galerkin method combined with the fixed point theorem, we show, in Section 2, the existence and uniqueness of a local in time solution. Secondly, under the smallness assumption on the initial data, we show, in Section 3, that the solution continues to exist globally in time. On the other hand, in Section 4, we prove that under some restrictions on the initial data and if the interior source dominates the boundary damping then the $L^{p}$-norm of the solution grows as an exponential function. Lastly, in Section 5 , we investigate the case when $\alpha=0$ and we prove that the solution ceases to exist and blows up in finite time.

\section{Preliminary and local existence}

In this section, we introduce some notations used throughout this paper. We also prove a local existence result of the solution of problem (1).

We denote

$$
H_{\Gamma_{0}}^{1}(\Omega)=\left\{u \in H^{1}(\Omega) / u_{\Gamma_{0}}=0\right\} .
$$


By $(.,$.$) we denote the scalar product in L^{2}(\Omega)$ i.e. $(u, v)(t)=\int_{\Omega} u(x, t) v(x, t) d x$. Also we mean by $\|\cdot\|_{q}$ the $L^{q}(\Omega)$ norm for $1 \leq q \leq \infty$, and by $\|\cdot\|_{q, \Gamma_{1}}$ the $L^{q}\left(\Gamma_{1}\right)$ norm.

Let $T>0$ be a real number and $X$ a Banach space endowed with norm $\|\cdot\|_{X} \cdot L^{p}(0, T ; X), 1 \leq$ $p<\infty$ denotes the space of functions $f$ which are $L^{p}$ over $(0, T)$ with values in $X$, which are measurable and $\|f\|_{X} \in L^{p}(0, T)$. This space is a Banach space endowed with the norm

$$
\|f\|_{L^{p}(0, T ; X)}=\left(\int_{0}^{T}\|f\|_{X}^{p} d t\right)^{1 / p} .
$$

$L^{\infty}(0, T ; X)$ denotes the space of functions $\left.f:\right] 0, T\left[\rightarrow X\right.$ which are measurable and $\|f\|_{X} \in$ $L^{\infty}(0, T)$. This space is a Banach space endowed with the norm:

$$
\|f\|_{L^{\infty}(0, T ; X)}=\operatorname{ess} \sup _{0<t<T}\|f\|_{X}
$$

We recall that if $X$ and $Y$ are two Banach spaces such that $X \hookrightarrow Y$ (continuous embedding), then

$$
L^{p}(0, T ; X) \hookrightarrow L^{p}(0, T ; Y), 1 \leq p \leq \infty .
$$

We will also use the embedding (see [1, Therorem 5.8]):

$$
H_{\Gamma_{0}}^{1}(\Omega) \hookrightarrow L^{p}(\Omega), 2 \leq p \leq \bar{p} \quad \text { where } \quad \bar{p}= \begin{cases}\frac{2 N}{N-2} & \text { if } N \geq 3, \\ +\infty & \text { if } N=1,2\end{cases}
$$

and also

$$
H_{\Gamma_{0}}^{1}(\Omega) \hookrightarrow L^{q}\left(\Gamma_{1}\right), 2 \leq q \leq \bar{q} \quad \text { where } \quad \bar{q}= \begin{cases}\frac{2(N-1)}{N-2} & \text { if } N \geq 3 \\ +\infty & \text { if } N=1,2 .\end{cases}
$$

For $2 \leq m \leq \bar{q}$, let us denote $V=H_{\Gamma_{0}}^{1}(\Omega) \cap L^{m}\left(\Gamma_{1}\right)$.

We assume that the relaxation functions $g$ is of class $C^{1}$ on $\mathbb{R}$ and satisfies:

$$
\forall s \in \mathbb{R}, g(s) \geq 0, \text { and }\left(1-\int_{0}^{\infty} g(s) d s\right)=l>0 .
$$

Moreover, we suppose that:

$$
\forall s \geq 0, g^{\prime}(s) \leq 0 .
$$

The hypotheses on the function $h$ are the following:

(H1) $h$ is continuous and strongly monotone, i.e. for $2 \leq m \leq \bar{q}$, there exists a constant $m_{0}>0$ such that

$$
(h(s)-h(v))(s-v) \geq m_{0}|s-v|^{m},
$$

(H2) there exist two positive constants $c_{m}$ and $C_{m}$ such that

$$
c_{m}|s|^{m} \leq h(s) s \leq C_{m}|s|^{m}, \quad \forall s \in \mathbb{R} .
$$


For a function $u \in C\left([0, T], H_{\Gamma_{0}}^{1}(\Omega)\right)$, let us introduce the following notation:

$$
(g \diamond u)(t)=\int_{0}^{t} g(t-s)\|\nabla u(s)-\nabla u(t)\|_{2}^{2} d s .
$$

Thus, when $u \in C\left([0, T], H_{\Gamma_{0}}^{1}(\Omega)\right) \cap C^{1}\left([0, T], L^{2}(\Omega)\right)$ such that $u_{t} \in L^{2}\left(0, T ; H_{\Gamma_{0}}^{1}(\Omega)\right)$, we have:

$$
\begin{aligned}
\frac{d}{d t}(g \diamond u)(t)= & \int_{0}^{t} g^{\prime}(t-s)\|\nabla u(s)-\nabla u(t)\|_{2}^{2} d s \\
& +\frac{d}{d t}\left(\|\nabla u(t)\|_{2}^{2}\right) \int_{0}^{t} g(s) d s-2 \int_{\Omega} \int_{0}^{t} g(t-s) \nabla u(s) \nabla u_{t}(t) d s d x \\
= & \left(g^{\prime} \diamond u\right)(t)-2 \int_{\Omega} \int_{0}^{t} g(t-s) \nabla u(s) \nabla u_{t}(t) d s d x \\
& +\frac{d}{d t}\left\{\|\nabla u(t)\|_{2}^{2} \int_{0}^{t} g(s) d s\right\}-g(t)\|\nabla u(t)\|_{2}^{2} .
\end{aligned}
$$

This last identity implies:

$$
\begin{aligned}
\int_{\Omega} \int_{0}^{t} g(t-s) \nabla u(s) \nabla u_{t}(t) d s d x= & \frac{1}{2}\left(g^{\prime} \diamond u\right)(t)+\frac{1}{2} \frac{d}{d t}\left\{\|\nabla u(t)\|_{2}^{2} \int_{0}^{t} g(s) d s\right\} \\
& -\frac{1}{2} g(t)\|\nabla u(t)\|_{2}^{2}-\frac{1}{2} \frac{d}{d t}(g \diamond u)(t) .
\end{aligned}
$$

For $u \in C\left([0, T], H_{\Gamma_{0}}^{1}(\Omega)\right) \cap C^{1}\left([0, T], L^{2}(\Omega)\right)$ such that $u_{t} \in L^{2}\left(0, T ; H_{\Gamma_{0}}^{1}(\Omega)\right)$, let us define the modified energy functional $E$ by:

$$
\begin{aligned}
E\left(t, u, u_{t}\right)=E(t)= & \frac{1}{2}\left\|u_{t}(t)\right\|_{2}^{2}+\frac{1}{2}\left\|u_{t}(t)\right\|_{2, \Gamma_{1}}^{2}+\frac{1}{2}\left(1-\int_{0}^{t} g(s) d s\right)\|\nabla u(t)\|_{2}^{2} \\
& +\frac{1}{2}(g \diamond u)(t)-\frac{1}{p}\|u(t)\|_{p}^{p} .
\end{aligned}
$$

The following local existence result of the solution of problem (1) is closely related to the one we have proved for a slightly different problem in [11, Theorem 2.1], where no memory term was present. Let us sate it:

Theorem 2.1. Assume that (5), (6) and (7) hold. Let $2 \leq p \leq \bar{q}$ and $\max \left(2, \frac{\bar{q}}{\bar{q}+1-p}\right) \leq m \leq \bar{q}$. Then given $u_{0} \in H_{\Gamma_{0}}^{1}(\Omega)$ and $u_{1} \in L^{2}(\Omega)$, there exists $T>0$ and a unique solution $u$ of the problem (1) on $(0, T)$ such that

$$
\begin{aligned}
u & \in C\left([0, T], H_{\Gamma_{0}}^{1}(\Omega)\right) \cap C^{1}\left([0, T], L^{2}(\Omega)\right), \\
u_{t} & \in L^{2}\left(0, T ; H_{\Gamma_{0}}^{1}(\Omega)\right) \cap L^{m}\left((0, T) \times \Gamma_{1}\right) .
\end{aligned}
$$

Let us mention that Theorem 2.1 also holds for $\alpha=0$. The proof of Theorem 2.1 can be done along the same line as in [11, Theorem 2.1]. The main idea of the proof is based on the combination 
between the Fadeo-Galerkin approximations and the contraction mapping theorem. However, for the convenience of the reader we give only the outline of the proof here.

For $u \in C\left([0, T], H_{\Gamma_{0}}^{1}(\Omega)\right) \cap C^{1}\left([0, T], L^{2}(\Omega)\right)$ given, let us consider the following problem:

$$
\begin{cases}v_{t t}-\Delta v-\alpha \Delta v_{t}+\int_{0}^{t} g(t-s) \Delta v(s) d s=|u|^{p-2} u, & x \in \Omega, t>0, \\ v(x, t)=0, & x \in \Gamma_{0}, t>0, \\ v_{t t}(x, t)=-\left[\frac{\partial v}{\partial \nu}(x, t)-\int_{0}^{t} g(t-s) \frac{\partial v}{\partial \nu}(x, s) d s+\frac{\alpha \partial v_{t}}{\partial \nu}(x, t)+h\left(v_{t}\right)\right] & x \in \Gamma_{1}, t>0, \\ v(x, 0)=u_{0}(x), v_{t}(x, 0)=u_{1}(x) & x \in \Omega .\end{cases}
$$

Definition 2.2. A function $v(x, t)$ such that

$$
\begin{aligned}
v & \in L^{\infty}\left(0, T ; H_{\Gamma_{0}}^{1}(\Omega)\right), \\
v_{t} & \in L^{2}\left(0, T ; H_{\Gamma_{0}}^{1}(\Omega)\right) \cap L^{m}\left((0, T) \times \Gamma_{1}\right), \\
v_{t} & \in L^{\infty}\left(0, T ; H_{\Gamma_{0}}^{1}(\Omega)\right) \cap L^{\infty}\left(0, T ; L^{2}\left(\Gamma_{1}\right)\right), \\
v_{t t} & \in L^{\infty}\left(0, T ; L^{2}(\Omega)\right) \cap L^{\infty}\left(0, T ; L^{2}\left(\Gamma_{1}\right)\right), \\
v(x, 0) & =u_{0}(x), \\
v_{t}(x, 0) & =u_{1}(x),
\end{aligned}
$$

is a generalized solution to the problem (12) if for any function $\omega \in H_{\Gamma_{0}}^{1}(\Omega) \cap L^{m}\left(\Gamma_{1}\right)$ and $\varphi \in$ $C^{1}(0, T)$ with $\varphi(T)=0$, we have the following identity:

$$
\begin{aligned}
\int_{0}^{T}\left(|u|^{p-2} u, w\right)(t) \varphi(t) d t & =\int_{0}^{T}\left[\left(v_{t t}, w\right)(t)+(\nabla v, \nabla w)(t)-\int_{0}^{t} g(t-s)(\nabla v(s), \nabla w(t)) d s\right. \\
& \left.+\alpha\left(\nabla v_{t}, \nabla w\right)(t) \varphi(t) d t\right]+\int_{0}^{T} \varphi(t)\left(\int_{\Gamma_{1}} v_{t t}(t) w d \Gamma+h\left(v_{t}\right) d \Gamma\right) d t
\end{aligned}
$$

Lemma 2.3. Let $2 \leq p \leq \bar{q}$ and $2 \leq m \leq \bar{q}$. Let $u_{0} \in H^{2}(\Omega) \cap V, u_{1} \in H^{2}(\Omega)$, then for any $T>0$, there exists a unique generalized solution (in the sense of Definition 2.2), $v(t, x)$ of problem (12).

The proof of Lemma 2.3 is essentially based on the Fadeo-Galerkin approximations combined with the compactness method and can be done along the same line as in [11, Lemma 2.2], we omit the details.

In the following lemma we state a local existence result of problem (12).

Lemma 2.4. Let $2 \leq p \leq \bar{q}$ and $\max \left(2, \frac{\bar{q}}{\bar{q}+1-p}\right) \leq m \leq \bar{q}$. Then given $u_{0} \in H_{\Gamma_{0}}^{1}(\Omega), u_{1} \in L^{2}(\Omega)$ there exists $T>0$ and a unique solution $v$ of the problem (12) on $(0, T)$ such that

$$
\begin{gathered}
v \in C\left([0, T], H_{\Gamma_{0}}^{1}(\Omega)\right) \cap C^{1}\left([0, T], L^{2}(\Omega)\right), \\
v_{t} \in L^{2}\left(0, T ; H_{\Gamma_{0}}^{1}(\Omega)\right) \cap L^{m}\left((0, T) \times \Gamma_{1}\right)
\end{gathered}
$$


and satisfies the energy inequality:

$$
\begin{aligned}
& \frac{1}{2}\left[\left\|u_{t}(t)\right\|_{2}^{2}+\left\|u_{t}(t)\right\|_{2, \Gamma_{1}}^{2}+\left(1-\int_{0}^{t} g(s) d s\right)\|\nabla u(t)\|_{2}^{2}+(g \diamond u)(t)\right]_{s}^{t} \\
& +\alpha \int_{s}^{t}\left\|\nabla v_{t}(\tau)\right\|_{2}^{2} d \tau+\int_{s}^{t} \int_{\Gamma_{1}} h\left(v_{t}(\sigma, \tau)\right) d \sigma d \tau \\
\leq & \int_{s}^{t} \int_{\Omega}|u(\tau)|^{p-2} u(\tau) v_{t}(\tau) d \tau d x
\end{aligned}
$$

for $0 \leq s \leq t \leq T$.

Proof. We first approximate $u \in C\left([0, T], H_{\Gamma_{0}}^{1}(\Omega)\right) \cap C^{1}\left([0, T], L^{2}(\Omega)\right)$ endowed with the standard norm $\|u\|=\max _{t \in[0, T]}\left\|u_{t}(t)\right\|_{2}+\|u(t)\|_{H^{1}(\Omega)}$, by a sequence $\left(u^{k}\right)_{k \in \mathbb{N}} \subset C^{\infty}([0, T] \times \bar{\Omega})$ by a standard convolution arguments (see [4]). Next, we approximate the initial data $u_{1} \in L^{2}(\Omega)$ by a sequence $\left(u_{1}^{k}\right)_{k \in \mathbb{N}} \subset C_{0}^{\infty}(\Omega)$. Finally, since the space $H^{2}(\Omega) \cap V \cap H_{\Gamma_{0}}^{1}(\Omega)$ is dense in $H_{\Gamma_{0}}^{1}(\Omega)$ for the $H^{1}$ norm, we approximate $u_{0} \in H_{\Gamma_{0}}^{1}(\Omega)$ by a sequence $\left(u_{0}^{k}\right)_{k \in \mathbb{N}} \subset H^{2}(\Omega) \cap V \cap H_{\Gamma_{0}}^{1}(\Omega)$.

We consider now the set of the following problems:

$$
\begin{cases}v_{t t}^{k}-\Delta v^{k}-\alpha \Delta v_{t}^{k}+\int_{0}^{t} g(t-s) \Delta v^{k}(s) d s=\left|u^{k}\right|^{p-2} u^{k}, & x \in \Omega, t>0, \\ v^{k}(x, t)=0, & x \in \Gamma_{0}, t>0, \\ v_{t t}^{k}(x, t)=-\left[\frac{\partial v^{k}}{\partial \nu}(x, t)-\int_{0}^{t} g(t-s) \frac{\partial v^{k}}{\partial \nu}(x, s) d s+\frac{\alpha \partial v_{t}^{k}}{\partial \nu}(x, t)+h\left(v_{t}^{k}\right)\right], & x \in \Gamma_{1}, t>0, \\ v^{k}(x, 0)=u_{0}^{k}, v_{t}^{k}(x, 0)=u_{1}^{k}, & x \in \Omega .\end{cases}
$$

Since every hypothesis of Lemma 2.3 are verified, we can find a sequence of unique solution $\left(v_{k}\right)_{k \in \mathbb{N}}$ of the problem (13). Our goal now is to show that $\left(v^{k}, v_{t}^{k}\right)_{k \in \mathbb{N}}$ is a Cauchy sequence in the space

$$
Y_{T}=\left\{\begin{array}{c}
\left(v, v_{t}\right) / v \in C\left([0, T], H_{\Gamma_{0}}^{1}(\Omega)\right) \cap C^{1}\left([0, T], L^{2}(\Omega)\right), \\
\left.v_{t} \in L^{2}\left(0, T ; H_{\Gamma_{0}}^{1}(\Omega)\right) \cap L^{m}\left((0, T) \times \Gamma_{1}\right)\right\}
\end{array}\right.
$$

endowed with the norm

$$
\left\|\left(v, v_{t}\right)\right\|_{Y_{T}}^{2}=\max _{0 \leq t \leq T}\left[\left\|v_{t}\right\|_{2}^{2}+l\|\nabla v\|_{2}^{2}\right]+\left\|v_{t}\right\|_{L^{m}\left((0, T) \times \Gamma_{1}\right)}^{2}+\int_{0}^{t}\left\|\nabla v_{t}(s)\right\|_{2}^{2} d s .
$$

For this purpose, we set $U=u^{k}-u^{k^{\prime}}, V=v^{k}-v^{k^{\prime}}$. It is straightforward to see that $V$ satisfies:

$$
\begin{cases}V_{t t}-\Delta V-\alpha \Delta V_{t}+\int_{0}^{t} g(t-s) \Delta V(s) d s=\left|u^{k}\right|^{p-2} u^{k}-\left|u^{k^{\prime}}\right|^{p-2} u^{k^{\prime}} & x \in \Omega, t>0, \\ V(x, t)=0 & x \in \Gamma_{0}, t>0, \\ V_{t t}(x, t)=-\left[\frac{\partial V}{\partial \nu}(x, t)-\int_{0}^{t} g(t-s) \frac{\partial V}{\partial \nu}(x, s) d s+\frac{\alpha \partial V_{t}}{\partial \nu}(x, t)+h\left(v_{t}^{k}\right)-h\left(v_{t}^{k^{\prime}}\right)\right] & x \in \Gamma_{1}, t>0, \\ V(x, 0)=u_{0}^{k}-u_{0}^{k^{\prime}}, V_{t}(x, 0)=u_{1}^{k}-u_{1}^{k^{\prime}} & x \in \Omega .\end{cases}
$$


We multiply the above differential equations by $V_{t}$, we integrate over $(0, t) \times \Omega$, we use integration by parts and the identity (9) to obtain:

$$
\begin{aligned}
& \frac{1}{2}\left(\left\|V_{t}(t)\right\|_{2}^{2}+\left\|V_{t}(t)\right\|_{2, \Gamma_{1}}^{2}+\left(1-\int_{0}^{t} g(r) d r\right)\|\nabla V(t)\|_{2}^{2}\right) \\
& +\alpha \int_{0}^{t}\left\|\nabla V_{t}\right\|_{2}^{2} d s+\int_{0}^{t} \int_{\Gamma_{1}}\left(h\left(v_{t}^{k}(x, \tau)\right)-h\left(v_{t}^{k^{\prime}}(x, \tau)\right)\right)\left(v_{t}^{k}(x, \tau)-v_{t}^{k^{\prime}}(x, \tau)\right) d \Gamma d \tau \\
& -\frac{1}{2} \int_{0}^{t}\left(g^{\prime} \diamond V\right)(s) d s+\frac{1}{2} \int_{0}^{t} g(s)\|\nabla V(s)\|_{2}^{2} d s \\
= & \frac{1}{2}\left(\left\|V_{t}(0)\right\|_{2}^{2}+\|\nabla V(0)\|_{2}^{2}+\left\|V_{t}(0)\right\|_{2, \Gamma_{1}}^{2}\right) \\
& +\int_{0}^{t} \int_{\Omega}\left(\left|u^{k}\right|^{p-2} u^{k}-\left|u^{k^{\prime}}\right|^{p-2} u^{k^{\prime}}\right)\left(v_{t}^{k}-v_{t}^{k^{\prime}}\right) d x d s, \quad \forall t \in(0, T) .
\end{aligned}
$$

Consequently, the above inequality together with (5), (6) and (7) gives

$$
\begin{aligned}
& \frac{1}{2}\left(\left\|V_{t}(t)\right\|_{2}^{2}+\left\|V_{t}(t)\right\|_{2, \Gamma_{1}}^{2}+l\|\nabla V(t)\|_{2}^{2}\right)+\alpha \int_{0}^{t}\left\|\nabla V_{t}\right\|_{2}^{2} d s+m_{0} \int_{0}^{t}\left\|V_{t}\right\|_{m, \Gamma_{1}}^{m} d s \\
\leq & \frac{1}{2}\left(\left\|V_{t}(0)\right\|_{2}^{2}+\|\nabla V(0)\|_{2}^{2}+\left\|V_{t}(0)\right\|_{2, \Gamma_{1}}^{2}\right) \\
& +\int_{0}^{t} \int_{\Omega}\left(\left|u^{k}\right|^{p-2} u^{k}-\left|u^{k^{\prime}}\right|^{p-2} u^{k^{\prime}}\right)\left(v_{t}^{k}-v_{t}^{k^{\prime}}\right) d x d s, \quad \forall t \in(0, T) .
\end{aligned}
$$

Following the same method as in [11], we deduce that there exists $C$ depending only on $\Omega$ and $p$ such that:

$$
\|V\|_{Y_{T}} \leq C\left(\left\|V_{t}(0)\right\|_{2}^{2}+\|\nabla V(0)\|_{2}^{2}+\left\|V_{t}(0)\right\|_{2, \Gamma_{1}}^{2}\right)+C T\|U\|_{Y_{T}}
$$

Since $\left(u_{0}^{k}\right)_{k \in \mathbb{N}}$ is a converging sequence in $H_{\Gamma_{0}}^{1}(\Omega),\left(u_{1}^{k}\right)_{k \in \mathbb{N}}$ is a converging sequence in $L^{2}(\Omega)$ and $\left(u^{k}\right)_{k \in \mathbb{N}}$ is a converging sequence in $C\left([0, T], H_{\Gamma_{0}}^{1}(\Omega)\right) \cap C^{1}\left([0, T], L^{2}(\Omega)\right)$ (so in $Y_{T}$ also), we conclude that $\left(v^{k}, v_{t}^{k}\right)_{k \in \mathbb{N}}$ is a Cauchy sequence in $Y_{T}$. Thus $\left(v^{k}, v_{t}^{k}\right)$ converges to a limit $\left(v, v_{t}\right) \in Y_{T}$. Now by the same procedure used by Georgiev and Todorova in [10], we prove that this limit is a weak solution of the problem (12). This completes the proof of the Lemma 2.4.

Proof of Theorem 2.1. In order to prove Theorem 2.1, we use the contraction mapping theorem. Indeed, for $T>0$, let us define the convex closed subset of $Y_{T}$ :

$$
X_{T}=\left\{\left(v, v_{t}\right) \in Y_{T} \text { such that } v(0)=u_{0}, v_{t}(0)=u_{1}\right\} .
$$

Let us denote:

$$
B_{R}\left(X_{T}\right)=\left\{v \in X_{T} ;\|v\|_{Y_{T}} \leq R\right\},
$$

the ball of radius $R$ in $X_{T}$. Then, Lemma 2.4 implies that for any $u \in X_{T}$, we may define $v=\Phi(u)$ the unique solution of (12) corresponding to $u$. Our goal now is to show that for a suitable $T>0$, $\Phi$ is a contractive map satisfying $\Phi\left(B_{R}\left(X_{T}\right)\right) \subset B_{R}\left(X_{T}\right)$. 
Let $u \in B_{R}\left(X_{T}\right)$ and $v=\Phi(u)$. Then for all $t \in[0, T]$ we have as in (15):

$$
\begin{aligned}
& \left\|v_{t}\right\|_{2}^{2}+l\|\nabla v\|_{2}^{2}+\left\|v_{t}\right\|_{2, \Gamma_{1}}^{2}+2 \alpha \int_{0}^{t}\left\|\nabla v_{t}\right\|_{2}^{2} d s+c \int_{0}^{t}\left\|v_{t}\right\|_{m, \Gamma_{1}}^{m} d s \\
& \leq\left\|u_{1}\right\|_{2}^{2}+\left\|\nabla u_{0}\right\|_{2}^{2}+\left\|u_{1}\right\|_{2, \Gamma_{1}}^{2}+2 \int_{0}^{t} \int_{\Omega}|u(\tau)|^{p-2} u(\tau) v_{t}(\tau) d x d \tau .
\end{aligned}
$$

Using Hölder's inequality, we can control the last term in the right hand side of the inequality (17) as follows:

$$
\int_{0}^{t} \int_{\Omega}|u(\tau)|^{p-2} u(\tau) v_{t}(\tau) d x d \tau \leq \int_{0}^{t}\|u(\tau)\|_{2 N /(N-2)}^{p-1}\left\|v_{t}(\tau)\right\|_{2 N /(3 N-N p+2(p-1))} d \tau
$$

Since $p \leq \frac{2 N}{N-2}$, we have:

$$
\frac{2 N}{(3 N-N p+2(p-1))} \leq \frac{2 N}{N-2}
$$

Thus, by Young's and Sobolev's inequalities, we get for all $\delta>0$ there exists $C(\delta)>0$, such that for all $t \in(0, T)$

$$
\int_{0}^{t} \int_{\Omega}|u(\tau)|^{p-2} u(\tau) v_{t}(\tau) d x d \tau \leq C(\delta) t R^{2(p-1)}+\delta \int_{0}^{t}\left\|\nabla v_{t}(\tau)\right\|_{2}^{2} d \tau .
$$

Inserting the last estimate in the inequality (17) and choosing $\delta$ small enough such that:

$$
\|v\|_{Y_{T}}^{2} \leq \frac{1}{2} R^{2}+C T R^{2(p-1)}
$$

Thus, for $T$ sufficiently small, we have $\|v\|_{Y_{T}} \leq R$. This shows that $v \in B_{R}\left(X_{T}\right)$.

To prove that $\Phi$ is a contraction, we have to follow the same steps (up to minor changes) as in [11]. We omit the details. Thus the proof of Theorem 2.1 is finished.

Remark 2.5. Let us say that the hypothesis on $m, \max \left(2, \frac{\bar{q}}{\bar{q}+1-p}\right) \leq m \leq \bar{q}$, is made to pass to the limit in the nonlinear term, by the same way we have used in [11, Equation (2.28)].

\section{Global existence}

In this section, we show that, under some restrictions on the initial data, the local solution of problem (1) can be continued in time and the lifespan of the solution will be $[0, \infty)$.

Definition 3.1. Let $2 \leq p \leq \bar{q}, \max \left(2, \frac{\bar{q}}{\bar{q}+1-p}\right) \leq m \leq \bar{q}, u_{0} \in H_{\Gamma_{0}}^{1}(\Omega)$ and $u_{1} \in L^{2}(\Omega)$. We denote by $u$ the solution of (1). We define:

$$
T_{\max }=\sup \{T>0, u=u(t) \text { exists on }[0, T]\} .
$$


Since the solution $u \in Y_{T}$ (the solution is "regular enough"), from the definition of the norm given by (14), let us recall that if $T_{\max }<\infty$, then

$$
\lim _{\substack{t \rightarrow T_{\max } \\ t<T_{\max }}}\|\nabla u(t)\|_{2}+\left\|u_{t}(t)\right\|_{2}=+\infty .
$$

If $T_{\max }<\infty$, we say that the solution of (1) blows up and that $T_{\max }$ is the blow up time. If $T_{\max }=\infty$, we say that the solution of (1) is global.

In order to study the blow up phenomenon or the global existence of the solution of (1), for all $0 \leq t<T_{\max }$, we define:

$$
\begin{aligned}
& I(t)=I(u(t))=\left(1-\int_{0}^{t} g(s) d s\right)\|\nabla u(t)\|_{2}^{2}+(g \diamond u)(t)-\|u\|_{p}^{p}, \\
& J(t)=J(u(t))=\frac{1}{2}\left(1-\int_{0}^{t} g(s) d s\right)\|\nabla u(t)\|_{2}^{2}+\frac{1}{2}(g \diamond u)(t)-\frac{1}{p}\|u\|_{p}^{p} .
\end{aligned}
$$

Thus the energy functional defined in (11) can be rewritten as

$$
E(u(t))=E(t)=J(t)+\frac{1}{2}\left\|u_{t}\right\|_{2}^{2}+\frac{1}{2}\left\|u_{t}\right\|_{2, \Gamma_{1}}^{2} .
$$

As in $[9,27]$, we denote by $B$ the best constant in the Poincaré-Sobolev embedding $H_{\Gamma_{0}}^{1}(\Omega) \hookrightarrow L^{p}(\Omega)$ defined by:

$$
B^{-1}=\inf \left\{\|\nabla u\|_{2}: u \in H_{\Gamma_{0}}^{1}(\Omega),\|u\|_{p}=1\right\} .
$$

For $u_{0} \in H_{\Gamma_{0}}^{1}(\Omega), u_{1} \in L^{2}(\Omega)$, we define:

$$
E(0)=\frac{1}{2}\left\|u_{1}\right\|_{2}^{2}+\frac{1}{2}\left\|u_{1}\right\|_{2, \Gamma_{1}}^{2}+\frac{1}{2}\left\|\nabla u_{0}\right\|_{2}^{2}-\frac{1}{p}\left\|u_{0}\right\|_{p}^{p} .
$$

The first goal is to prove that the above energy $E(t)$ defined in (11) is a non-increasing function along the trajectories. More precisely, we have the following result:

Lemma 3.2. Let $2 \leq p \leq \bar{q}, \max \left(2, \frac{\bar{q}}{\bar{q}+1-p}\right) \leq m \leq \bar{q}$, and $u$ be the solution of (1). Then, for all $t>0$, we have

$$
\begin{aligned}
\frac{d E(t)}{d t} & =\frac{1}{2}\left(g^{\prime} \diamond u\right)(t)-\frac{1}{2} g(t)\|\nabla u(t)\|_{2}^{2}-\alpha\left\|\nabla u_{t}\right\|_{2}^{2}-\int_{\Gamma_{1}} h\left(u_{t}\right) u_{t} d \Gamma \\
& \leq \frac{1}{2}\left(g^{\prime} \diamond u\right)(t)-\alpha\left\|\nabla u_{t}\right\|_{2}^{2}-\int_{\Gamma_{1}} h\left(u_{t}\right) u_{t} d \Gamma, \quad \forall t \in\left[0, T_{\max }\right) .
\end{aligned}
$$

Proof. Multiplying the first equation in (1) by $u_{t}$, integrating over $\Omega$, using integration by parts we get:

$$
\begin{aligned}
& \frac{d}{d t}\left\{\frac{1}{2}\left\|u_{t}\right\|_{2}^{2}+\frac{1}{2}\left\|u_{t}\right\|_{2, \Gamma_{1}}^{2}+\frac{1}{2}\|\nabla u\|_{2}^{2}-\frac{1}{p}\|u\|_{p}^{p}\right\} \\
& -\int_{\Omega} \int_{0}^{t} g(t-s) \nabla u(s) \nabla u_{t}(t) d s d x \\
& =-\alpha\left\|\nabla u_{t}\right\|_{2}^{2}-\int_{\Gamma_{1}} h\left(u_{t}\right) u_{t} d \Gamma .
\end{aligned}
$$

A simple use of the identity (10) gives (22). This completes the proof of Lemma 3.2. 
Lemma 3.3. Let $2 \leq p \leq \bar{q}, \max \left(2, \frac{\bar{q}}{\bar{q}+1-p}\right) \leq m \leq \bar{q}$. Assume that (5) and (6) hold. Then given $u_{0} \in H_{\Gamma_{0}}^{1}(\Omega), u_{1} \in L^{2}(\Omega)$ satisfying

$$
\left\{\begin{array}{l}
\beta=\frac{B^{p}}{l}\left(\frac{2 p}{l(p-2)} E(0)^{(p-2) / 2}\right)<1 \\
I\left(u_{0}\right)>0
\end{array}\right.
$$

we have:

$$
I(u(t))>0, \quad \forall t \in\left[0, T_{\max }\right) .
$$

Proof. Since $I\left(u_{0}\right)>0$, then by continuity, there exists $T^{*}<T_{\max }$, such that

$$
I(t)>0, \quad \forall t \in\left[0, T^{*}\right]
$$

which implies that for all $t \in\left[0, T^{*}\right]$,

$$
\begin{aligned}
J(t) & =\frac{1}{p} I(t)+\frac{p-2}{2 p}\left\{\left(1-\int_{0}^{t} g(s) d s\right)\|\nabla u(t)\|_{2}^{2}+(g \diamond u)(t)\right\} \\
& \geq \frac{p-2}{2 p}\left\{\left(1-\int_{0}^{t} g(s) d s\right)\|\nabla u(t)\|_{2}^{2}+(g \diamond u)(t)\right\} .
\end{aligned}
$$

By using (5), (6), (20) and (22), we easily get, for all $t \in\left[0, T^{*}\right]$

$$
\begin{aligned}
l\|\nabla u(t)\|_{2}^{2} & \leq \frac{2 p}{p-2} J(t), \\
& \leq \frac{2 p}{p-2} E(t) \leq \frac{2 p}{p-2} E(0) .
\end{aligned}
$$

From the definition of the constant $B$ in (21), we first get:

$$
\forall t \in\left[0, T^{*}\right],\|u(t)\|_{p}^{p} \leq B^{p}\|\nabla u(t)\|_{2}^{p} .
$$

Since we have:

$$
\forall t \in\left[0, T^{*}\right], B^{p}\|\nabla u(t)\|_{2}^{p}=\frac{B^{p}}{l}\|\nabla u(t)\|_{2}^{p-2}\left(l\|\nabla u(t)\|_{2}^{2}\right),
$$

by exploiting (26) and (24), we obtain, for all $t \in\left[0, T^{*}\right]$ :

$$
\begin{aligned}
\|u(t)\|_{p}^{p} & \leq \beta l\left(\|\nabla u(t)\|_{2}^{2}\right) \\
& \leq \beta\left(1-\int_{0}^{t} g(s) d s\right)\|\nabla u(t)\|_{2}^{2} \\
& <\left(1-\int_{0}^{t} g(s) d s\right)\|\nabla u(t)\|_{2}^{2} .
\end{aligned}
$$

Therefore, by using (18), we conclude that

$$
I(t)>0, \quad \forall t \in\left[0, T^{*}\right] .
$$

Using the fact that $E$ is decreasing along the trajectory, we get:

$$
\forall 0 \leq t<T_{\max }, \frac{B^{p}}{l}\left(\frac{2 p}{l(p-2)} E(t)\right)^{(p-2) / 2} \leq \beta<1 .
$$

By repeating this procedure, $T^{*}$ is extended to $T_{\max }$. 
Now, we are able to state the global existence theorem.

Theorem 3.4. Let $2 \leq p \leq \bar{q}, \max \left(2, \frac{\bar{q}}{\bar{q}+1-p}\right) \leq m \leq \bar{q}$. Assume that (5) and (6) hold. Then given $u_{0} \in H_{\Gamma_{0}}^{1}(\Omega), u_{1} \in L^{2}(\Omega)$ satisfying (24). Then the solution of (1) is global and bounded.

Proof. To prove Theorem 3.4, using the definition of $T_{m a x}$, we have just to check that

$$
\|\nabla u(t)\|_{2}^{2}+\left\|u_{t}(t)\right\|_{2}^{2}
$$

is uniformly bounded in time. To achieve this, we use (19), (20), (22) and (26) to get

$$
\begin{aligned}
E(0) & \geq E(t)=J(t)+\frac{1}{2}\left\|u_{t}\right\|_{2}^{2}+\frac{1}{2}\left\|u_{t}\right\|_{2, \Gamma_{1}}^{2} \\
& \geq \frac{p-2}{2 p}\|\nabla u(t)\|_{2}^{2}+\frac{1}{2}\left\|u_{t}(t)\right\|_{2}^{2} .
\end{aligned}
$$

Therefore,

$$
\|\nabla u(t)\|_{2}^{2}+\left\|u_{t}(t)\right\|_{2}^{2} \leq C E(0)
$$

where $C$ is a positive constant, which depends only on $p$.

\section{Exponential growth for $\alpha>0$}

In this section we will prove that when the initial data are large enough, the energy of the solution of problem (1) defined by (11) grows exponentially and thus so the $L^{p}$ norm.

In order to state and prove the exponential growth result, we introduce the following constants:

$$
B_{1}=\frac{B}{l}, \quad \alpha_{1}=B_{1}^{-p /(p-2)}, \quad E_{1}=\left(\frac{1}{2}-\frac{1}{p}\right) \alpha_{1}^{2}, \quad E_{2}=\left(\frac{l}{2}-\frac{1}{p}\right) \alpha_{1}^{2}
$$

Let us first mention that $E_{2}<E_{1}$.

The following Lemma will play an essential role in the proof of the exponential growth result, and it is inspired by the work in [7] where the authors proved a similar lemma for the wave equation.

First, we define the function

$$
\gamma(t):=\left(1-\int_{0}^{t} g(s) d s\right)\|\nabla u(t)\|_{2}^{2}+(g \diamond u)(t) .
$$

Let us rewrite the energy functional $E$ defined by (11) as:

$$
E(t)=\frac{1}{2}\left\|u_{t}(t)\right\|_{2}^{2}+\frac{1}{2}\left\|u_{t}(t)\right\|_{2, \Gamma_{1}}^{2}+\frac{1}{2} \gamma(t)-\frac{1}{p}\|u(t)\|_{p}^{p}
$$

Lemma 4.1. Let $2 \leq p \leq \bar{q}, \max \left(2, \frac{\bar{q}}{\bar{q}+1-p}\right) \leq m \leq \bar{q}$. Let $u$ be the solution of (1). Assume that

$$
E(0)<E_{1} \quad \text { and } \quad\left\|\nabla u_{0}\right\|_{2} \geq \alpha_{1} .
$$

Then there exists a constant $\alpha_{2}>\alpha_{1}$ such that

$$
(\gamma(t))^{1 / 2} \geq \alpha_{2}, \quad \forall t \in\left[0, T_{\max }\right)
$$

and

$$
\|u(t)\|_{p} \geq B_{1} \alpha_{2}, \quad \forall t \in\left[0, T_{\max }\right) .
$$


Proof. We first note that, by (30), we have:

$$
\begin{aligned}
E(t) & \geq \frac{1}{2} \gamma(t)-\frac{1}{p}\|u(t)\|_{p}^{p} \\
& \geq \frac{1}{2} \gamma(t)-\frac{B_{1}^{p}}{p}\left(l\|\nabla u(t)\|_{2}\right)^{p} \\
& \geq \frac{1}{2} \gamma(t)-\frac{B_{1}^{p}}{p}(\gamma(t))^{p / 2} \\
& =\frac{1}{2} \alpha^{2}-\frac{B_{1}^{p}}{p} \alpha^{p}=F(\alpha),
\end{aligned}
$$

where $\alpha=(\gamma(t))^{1 / 2}$. It is easy to verify that $F$ is increasing for $0<\alpha<\alpha_{1}$, decreasing for $\alpha>\alpha_{1}$, $F(\alpha) \rightarrow-\infty$ as $\alpha \rightarrow+\infty$, and

$$
F\left(\alpha_{1}\right)=E_{1},
$$

where $\alpha_{1}$ is given in (28). Therefore, since $E(0)<E_{1}$, there exists $\alpha_{2}>\alpha_{1}$ such that $F\left(\alpha_{2}\right)=$ $E(0)$.

If we set $\alpha_{0}=(\gamma(0))^{1 / 2}$, then by (34) we have:

$$
F\left(\alpha_{0}\right) \leq E(0)=F\left(\alpha_{2}\right),
$$

which implies that $\alpha_{0} \geq \alpha_{2}$.

Now to establish (32), we suppose by contradiction that:

$$
\left(\gamma\left(t_{0}\right)\right)^{1 / 2}<\alpha_{2},
$$

for some $t_{0}>0$ and by the continuity of $\gamma($.$) , we may choose t_{0}$ such that

$$
\left(\gamma\left(t_{0}\right)\right)^{1 / 2}>\alpha_{1}
$$

Using again (34) leads to:

$$
E\left(t_{0}\right) \geq F\left(\gamma\left(t_{0}\right)^{1 / 2}\right)>F\left(\alpha_{2}\right)=E(0) .
$$

But this is impossible since for all $t>0, E(t) \leq E(0)$. Hence (32) is established.

To prove (33), we use (30) to get:

$$
\frac{1}{2} \gamma(t) \leq E(0)+\frac{1}{p}\|u(t)\|_{p}^{p} .
$$

Consequently, using (32) leads to:

$$
\begin{aligned}
\frac{1}{p}\|u(t)\|_{p}^{p} & \geq \frac{1}{2} \gamma(t)-E(0) \\
& \geq \frac{1}{2} \alpha_{2}^{2}-E(0)
\end{aligned}
$$

But we have:

$$
\frac{1}{2} \alpha_{2}^{2}-E(0)=\frac{1}{2} \alpha_{2}^{2}-F\left(\alpha_{2}\right)=\frac{B_{1}^{p}}{p} \alpha_{2}^{p} .
$$

Therefore (33) holds. This finishes the proof of Lemma 4.1. 
The exponential growth result reads as follows:

Theorem 4.2. Suppose that (5), (6) (8) hold. Assume that

$$
2 \leq m \quad \text { and } \quad \max (m, 2 / l)<p \leq \bar{p} .
$$

Then, the solution of (1) satisfying

$$
E(0)<E_{2} \text { and }\left\|\nabla u_{0}\right\|_{2} \geq \alpha_{1},
$$

grows exponentially in the $L^{p}$ norm.

Remark 4.3. It is obvious that for $g=0$, we have $E_{1}=E_{2}$, and Theorem 4.2 reduces to Theorem 3.1 in [11].

Remark 4.4. In Theorem 4.2, the condition

$$
\int_{0}^{\infty} g(s) d s<\frac{(p / 2)-1}{(p / 2)-1+(1 / 2 p)}
$$

used in [16, 17, 21, 22, 23, 26, 28] is unnecessary and our result holds without it.

Remark 4.5. Let us denote $c_{1}=\left(l-\frac{2}{p}\right)-2 E_{2}\left(B_{1} \alpha_{2}\right)^{-p}$. Since we have seen that $\alpha_{2}>\alpha_{1}$, using the definition of $E_{2}$, we easily get $c_{1}>0$. This constant will play an important role in the proof of Theorem 4.2

Proof of Theorem 4.2. We implement the so-called Georgiev-Todorova method (see [10, 22] and also [20]). So, we suppose that the solution exists for all time and we will prove an exponential growth. For this purpose, we set:

$$
\mathscr{H}(t)=E_{2}-E(t) .
$$

Of course by (31) and (22) and since $E_{2}<E_{1}$, we deduce that $\mathscr{H}$ is a non-decreasing function. So, by using (30) and, (36) we get successively:

$$
0<\mathscr{H}(0) \leq \mathscr{H}(t) \leq E_{2}-E(t) \leq E_{1}-\frac{1}{2} \gamma(t)+\frac{1}{p}\|u(t)\|_{p}^{p} .
$$

On one hand as $F\left(\alpha_{1}\right)=E_{1}$ and $\forall t>0, \gamma(t) \geq \alpha_{2}^{2}>\alpha_{1}^{2}$, we obtain:

$$
E_{1}-\frac{1}{2} \gamma(t)<F\left(\alpha_{1}\right)-\frac{1}{2} \alpha_{1}^{2}
$$

On the other hand, since

$$
F\left(\alpha_{1}\right)-\frac{1}{2} \alpha_{1}^{2}=-\frac{B_{1}^{p}}{p} \alpha_{1}^{p},
$$

we obtain the following inequality:

$$
0<\mathscr{H}(0) \leq \mathscr{H}(t) \leq \frac{1}{p}\|u(t)\|_{p}^{p}, \quad \forall t \geq 0 .
$$


For $\varepsilon$ small to be chosen later, and inspired by the ideas of the authors in [11], we then define the auxiliary function:

$$
\mathscr{L}(t)=\mathscr{H}(t)+\varepsilon \int_{\Omega} u_{t} u d x+\varepsilon \int_{\Gamma_{1}} u_{t} u d \Gamma+\frac{\varepsilon \alpha}{2}\|\nabla u\|_{2}^{2} .
$$

Let us remark that $\mathscr{L}$ is a small perturbation of the energy. By taking the time derivative of (38), using problem (1), we obtain:

$$
\begin{aligned}
\frac{d \mathscr{L}(t)}{d t}= & \alpha\left\|\nabla u_{t}\right\|_{2}^{2}+\int_{\Gamma_{1}} h\left(u_{t}\right) u_{t} d \Gamma+\varepsilon\left\|u_{t}\right\|_{2}^{2}-\varepsilon\|\nabla u\|_{2}^{2} \\
& +\varepsilon\|u\|_{p}^{p}+\varepsilon\left\|u_{t}\right\|_{2, \Gamma_{1}}^{2}-\varepsilon \int_{\Gamma_{1}} h\left(u_{t}\right) u(x, t) d \sigma \\
& +\int_{\Omega} \nabla u(t) \int_{0}^{t} g(t-s) \nabla u(s) d s d x
\end{aligned}
$$

By making use of (8) and the following Young's inequality

$$
X Y \leq \frac{\lambda^{\mu} X^{\mu}}{\mu}+\frac{\lambda^{-\nu} Y^{\nu}}{\nu}
$$

$X, Y \geq 0, \lambda>0, \mu, \nu \in \mathbb{R}^{+}$such that $1 / \mu+1 / \nu=1$, then we get

$$
\begin{aligned}
\int_{\Gamma_{1}} h\left(u_{t}\right) u d \Gamma & \leq C_{m} \int_{\Gamma_{1}}\left|u_{t}\right|^{m-2} u_{t} u d \Gamma \\
& \leq C_{m} \frac{\lambda^{m}}{m}\|u\|_{m, \Gamma_{1}}^{m}+C_{m} \frac{m-1}{m} \lambda^{-m /(m-1)}\left\|u_{t}\right\|_{m, \Gamma_{1}}^{m} .
\end{aligned}
$$

Now, the term involving $g$ on the right-hand side of (39) can be written as

$$
\begin{aligned}
\int_{\Omega} \nabla u(t, x) \int_{0}^{t} g(t-s) \nabla u(s, x) d s d x=\|\nabla u(t)\|_{2}^{2}\left(\int_{0}^{t} g(s) d s\right) \\
+\int_{\Omega} \nabla u(t, x) \int_{0}^{t} g(t-s)(\nabla u(s, x)-\nabla u(t, x)) d s d x .
\end{aligned}
$$

On the other hand, by using Hölder's and Young's inequalities, we infer that for all $\mu>0$, we get

$$
\begin{aligned}
& \int_{\Omega} \nabla u(t, x) \int_{0}^{t} g(t-s)(\nabla u(s, x)-\nabla u(t, x)) d s d x \\
& \leq \int_{0}^{t} g(t-s)\|\nabla u(t)\|_{2}\|\nabla u(s)-\nabla u(t)\|_{2} d s \\
& \leq \mu(g \diamond u)(t)+\frac{1}{4 \mu}\left(\int_{0}^{t} g(s) d s\right)\|\nabla u(t)\|_{2}^{2} .
\end{aligned}
$$

Inserting the estimates (41) and (42) into (39), taking into account the inequality (43) and making use of (8), we obtain by choosing $\mu=1 / 2$ and multiplying by $l$

$$
\begin{aligned}
l \mathscr{L}^{\prime}(t) \geq & \alpha l\left\|\nabla u_{t}\right\|_{2}^{2}+l\left(c_{m}-C_{m} \varepsilon \frac{m-1}{m} \lambda^{-m /(m-1)}\right)\left\|u_{t}\right\|_{m, \Gamma_{1}}^{m}+\varepsilon l\left\|u_{t}\right\|_{2}^{2} \\
& +\varepsilon l\|u\|_{p}^{p}+\varepsilon l\left\|u_{t}\right\|_{2, \Gamma_{1}}^{2}-C_{m} \varepsilon l \frac{\lambda^{m}}{m}\|u\|_{m, \Gamma_{1}}^{m} \\
& -\frac{\varepsilon l}{2}(g \diamond u)(t)-\varepsilon l\|\nabla u(t)\|_{2}^{2} .
\end{aligned}
$$


We want now to estimate the term involving $\|u\|_{m, \Gamma_{1}}^{m}$ in (44). We proceed as in [11]. Then, we have

$$
\|u\|_{m, \Gamma_{1}} \leq C\|u\|_{H^{s}(\Omega)},
$$

which holds for:

$$
m \geq 1 \quad \text { and } \quad 0<s<1, \quad s \geq \frac{N}{2}-\frac{N-1}{m}>0,
$$

where $C$ here and in the sequel denotes a generic positive constant which may change from line to line.

Recalling the interpolation and Poincaré's inequalities (see [18])

$$
\begin{aligned}
\|u\|_{H^{s}(\Omega)} & \leq C\|u\|_{2}^{1-s}\|\nabla u\|_{2}^{s}, \\
& \leq C\|u\|_{p}^{1-s}\|\nabla u\|_{2}^{s},
\end{aligned}
$$

we finally have the following inequality:

$$
\|u\|_{m, \Gamma_{1}} \leq C\|u\|_{p}^{1-s}\|\nabla u\|_{2}^{s} .
$$

If $s<2 / m$, using again Young's inequality, we get:

$$
\|u\|_{m, \Gamma_{1}}^{m} \leq C\left[\left(\|u\|_{p}^{p}\right)^{\frac{m(1-s) \mu}{p}}+\left(\|\nabla u\|_{2}^{2}\right)^{\frac{m s \theta}{2}}\right]
$$

for $1 / \mu+1 / \theta=1$. Here we choose $\theta=2 / m s$, to get $\mu=2 /(2-m s)$. Therefore the previous inequality becomes:

$$
\|u\|_{m, \Gamma_{1}}^{m} \leq C\left[\left(\|u\|_{p}^{p}\right)^{\frac{m(1-s) 2}{(2-m s) p}}+\|\nabla u\|_{2}^{2}\right] .
$$

Now, choosing $s$ such that:

$$
0<s \leq \frac{2(p-m)}{m(p-2)}
$$

we get:

$$
\frac{2 m(1-s)}{(2-m s) p} \leq 1
$$

Once the inequality (48) is satisfied, we use the classical algebraic inequality:

$$
z^{\nu} \leq(z+1) \leq\left(1+\frac{1}{\omega}\right)(z+\omega), \quad \forall z \geq 0, \quad 0<\nu \leq 1, \quad \omega \geq 0
$$

to obtain the following estimate:

$$
\begin{aligned}
\left(\|u\|_{p}^{p}\right)^{\frac{m(1-s) 2}{(2-m s) p}} & \leq d\left(\|u\|_{p}^{p}+\mathscr{H}(0)\right) \\
& \leq d\left(\|u\|_{p}^{p}+\mathscr{H}(t)\right), \quad \forall t \geq 0,
\end{aligned}
$$

where we have set $d=1+1 / \mathscr{H}(0)$. Inserting the estimate (50) into (46) we obtain the following important inequality:

$$
\|u\|_{m, \Gamma_{1}}^{m} \leq C\left[\|u\|_{p}^{p}+l\|\nabla u\|_{2}^{2}+\mathscr{H}(t)\right]
$$


Keeping in mind that $l=1-\int_{0}^{\infty} g(s) d s$, in order to control the term $\|\nabla u\|_{2}^{2}$ in equation (44), we preferely use (as $\mathscr{H}(t)>0$ ), the following estimate:

$$
\|u\|_{m, \Gamma_{1}}^{m} \leq C\left[\|u\|_{p}^{p}+l\|\nabla u\|_{2}^{2}+2 \mathscr{H}(t)\right] .
$$

which gives finally:

$$
\begin{aligned}
\|u\|_{m, \Gamma_{1}}^{m} \leq & C\left[2 E_{2}+\left(1+\frac{2}{p}\right)\|u\|_{p}^{p}-\left\|u_{t}\right\|_{2}^{2}-\left\|u_{t}\right\|_{2, \Gamma_{1}}^{2}\right. \\
& \left.+\left(l-\left(1-\int_{0}^{t} g(s) d s\right)\right)\|\nabla u\|_{2}^{2}-(g \diamond u)(t)\right] .
\end{aligned}
$$

Since $1-\int_{0}^{t} g(s) d s \geq l$, then we obtain from above

$$
\|u\|_{m, \Gamma_{1}}^{m} \leq C\left[2 E_{2}+\left(1+\frac{2}{p}\right)\|u\|_{p}^{p}-\left\|u_{t}\right\|_{2}^{2}-\left\|u_{t}\right\|_{2, \Gamma_{1}}^{2}-(g \diamond u)(t)\right] .
$$

Now, inserting (53) into (44), then we infer that:

$$
\begin{aligned}
l \mathscr{L}^{\prime}(t) \geq & \alpha l\left\|\nabla u_{t}\right\|_{2}^{2}+l\left(c_{m}-C_{m} \varepsilon \frac{m-1}{m} \lambda^{-m /(m-1)}\right)\left\|u_{t}\right\|_{m, \Gamma_{1}}^{m} \\
& +\varepsilon\left(l+l C_{m} \frac{\lambda^{m}}{m} C\right)\left\|u_{t}\right\|_{2}^{2}+\varepsilon\left(l+l C_{m} \frac{\lambda^{m}}{m} C\right)\left\|u_{t}\right\|_{2, \Gamma_{1}}^{2} \\
& +\varepsilon\left\{l-C_{m} l \frac{\lambda^{m}}{m} C\left(1+\frac{2}{p}\right)\right\}\|u\|_{p}^{p} \\
& +\varepsilon\left(C_{m} l \frac{\lambda^{m}}{m} C-\frac{l}{2}\right)(g \diamond u)(t)-\varepsilon l\|\nabla u(t)\|_{2}^{2}-2 C_{m} \varepsilon l \frac{\lambda^{m}}{m} C E_{2} .
\end{aligned}
$$

From (36), we get

$$
\begin{aligned}
\mathscr{H}(t) \leq & E_{2}-\frac{1}{2}\left(1-\int_{0}^{t} g(s) d s\right)\|\nabla u(t)\|_{2}^{2} \\
& -\frac{1}{2}(g \diamond u)(t)+\frac{1}{p}\|u(t)\|_{p}^{p} \\
\leq & E_{2}-\frac{l}{2}\|\nabla u(t)\|_{2}^{2}-\frac{1}{2}(g \diamond u)(t)+\frac{1}{p}\|u(t)\|_{p}^{p} .
\end{aligned}
$$

This last inequality gives

$$
-l\|\nabla u(t)\|_{2}^{2} \geq 2\left(\mathscr{H}(t)-E_{2}+\frac{1}{2}(g \diamond u)(t)-\frac{1}{p}\|u(t)\|_{p}^{p}\right) .
$$

Consequently, (54) takes the form

$$
\begin{aligned}
l \mathscr{L}^{\prime}(t) \geq & \alpha l\left\|\nabla u_{t}\right\|_{2}^{2}+l\left(c_{m}-C_{m} \varepsilon \frac{m-1}{m} \lambda^{-m /(m-1)}\right)\left\|u_{t}\right\|_{m, \Gamma_{1}}^{m} \\
& +\varepsilon\left(l+l C_{m} \frac{\lambda^{m}}{m} C\right)\left\|u_{t}\right\|_{2}^{2}+\varepsilon\left(l+l C_{m} \frac{\lambda^{m}}{m} C\right)\left\|u_{t}\right\|_{2, \Gamma_{1}}^{2} \\
& +\varepsilon\left\{l-\frac{2}{p}-C_{m} l \frac{\lambda^{m}}{m} C\left(1+\frac{2}{p}\right)\right\}\|u\|_{p}^{p}-2 \varepsilon E_{2} \\
& +\varepsilon\left(C_{m} l \frac{\lambda^{m}}{m} C-\frac{l}{2}+1\right)(g \diamond u)(t)+2 \varepsilon \mathscr{H}(t)-2 C_{m} \varepsilon l \frac{\lambda^{m}}{m} C E_{2} .
\end{aligned}
$$


Now to estimate the terms involving $\|u\|_{p}^{p}$ and $E_{2}$ in (56), we simply write:

$$
\left(l-\frac{2}{p}\right)\|u\|_{p}^{p}-2 E_{2}=\left(l-\frac{2}{p}\right)\|u\|_{p}^{p}-2 E_{2} \frac{\|u\|_{p}^{p}}{\|u\|_{p}^{p}} .
$$

Then by using (33), we get:

$$
\left(l-\frac{2}{p}\right)\|u\|_{p}^{p}-2 E_{2} \geq c_{1}\|u\|_{p}^{p},
$$

where $c_{1}>0$ is defined in Remark 4.5. Thus, (56) becomes:

$$
\begin{aligned}
l \mathscr{L}^{\prime}(t) \geq & \alpha l\left\|\nabla u_{t}\right\|_{2}^{2}+l\left(c_{m}-C_{m} \varepsilon \frac{m-1}{m} \lambda^{-m /(m-1)}\right)\left\|u_{t}\right\|_{m, \Gamma_{1}}^{m} \\
& +\varepsilon\left(l+l C_{m} \frac{\lambda^{m}}{m} C\right)\left\|u_{t}\right\|_{2}^{2}+\varepsilon\left(l+l C_{m} \frac{\lambda^{m}}{m} C\right)\left\|u_{t}\right\|_{2, \Gamma_{1}}^{2} \\
& +\varepsilon\left\{c_{1}-C_{m} l \frac{\lambda^{m}}{m} C\left(1+\frac{2}{p}\right)-4 C_{m} \varepsilon l \frac{\lambda^{m}}{m} C E_{2}\left(B_{1} \alpha_{2}\right)^{-p}\right\}\|u\|_{p}^{p} \\
& +\varepsilon\left(C_{m} l \frac{\lambda^{m}}{m} C-\frac{l}{2}+1\right)(g \diamond u)(t)+2 \varepsilon\left(\mathscr{H}(t)+C_{m} l \frac{\lambda^{m}}{m} C E_{2}\right) .
\end{aligned}
$$

Notice that since $l<1$, we first have :

$$
\forall \lambda>0, C_{m} l \frac{\lambda^{m}}{m} C-\frac{l}{2}+1>0 .
$$

At this point, we pick $\lambda$ small enough such that:

$$
c_{1}-C_{m} l \frac{\lambda^{m}}{m} C\left(1+\frac{2}{p}\right)-4 C_{m} \varepsilon l \frac{\lambda^{m}}{m} C E_{2}\left(B_{1} \alpha_{2}\right)^{-p}>0 .
$$

Once $\lambda$ is fixed, we may choose $\varepsilon$ small enough such that

$$
\left\{\begin{array}{l}
c_{m}-C_{m} \varepsilon \frac{m-1}{m} \lambda^{-m /(m-1)}>0, \\
\mathscr{L}(0)>0 .
\end{array}\right.
$$

Consequently, we end up with the estimate:

$$
\mathscr{L}^{\prime}(t) \geq \eta_{1}\left(\left\|u_{t}\right\|_{2}^{2}+\left\|u_{t}\right\|_{2, \Gamma_{1}}^{2}+\|u\|_{p}^{p}+\mathscr{H}(t)+E_{2}\right), \quad \forall t \geq 0 .
$$

Next, it is clear that, by Young's and Poincaré's inequalities, we have:

$$
\mathscr{L}(t) \leq \gamma\left[\mathscr{H}(t)+\left\|u_{t}\right\|_{2}^{2}+\left\|u_{t}\right\|_{2, \Gamma_{1}}^{2}+\|\nabla u\|_{2}^{2}\right] \text { for some } \gamma>0 .
$$

Since $\mathscr{H}(t)>0$, then for all $t \geq 0$, we have:

$$
\frac{l}{2}\|\nabla u\|_{2}^{2} \leq \frac{1}{p}\|u\|_{p}^{p}+E_{2}
$$


Thus, the inequality (59) becomes:

$$
\mathscr{L}(t) \leq \zeta\left[\mathscr{H}(t)+\left\|u_{t}\right\|_{2}^{2}+\left\|u_{t}\right\|_{2, \Gamma_{1}}^{2}+\|u\|_{p}^{p}+E_{2}\right] \text { for some } \zeta>0 .
$$

From the two inequalities (58) and (61), we finally obtain the differential inequality:

$$
\frac{d \mathscr{L}(t)}{d t} \geq \mu \mathscr{L}(t) \text { for some } \mu>0 .
$$

An integration of the previous differential inequality (62) between 0 and $t$ gives the following estimate for the function $\mathscr{L}$ :

$$
\mathscr{L}(t) \geq \mathscr{L}(0) e^{\mu t} .
$$

On the other hand, from the definition of the function $\mathscr{L}$, from inequality (37) and for small values of the parameter $\varepsilon$, it follows that:

$$
\mathscr{L}(t) \leq \frac{1}{p}\|u\|_{p}^{p}
$$

From the two inequalities (63) and (64) we conclude the exponential growth of the solution in the $L^{p}$-norm.

\section{Blow up in finite time for $\alpha=0$}

In this section, we prove that in the absence of the strong damping $-\Delta u_{t}$, (i.e. $\alpha=0$ ), the solution of problem (1) blows up in finite time that is it exists $0<T^{*}<\infty$ such that $\|u(t)\|_{p} \rightarrow \infty$ as $t \rightarrow T^{*}$.

The blow up result reads as follows:

Theorem 5.1. Suppose that (5), (6) and (8) hold. Assume that

$$
2<m \text { and } \max (m, 2 / l)<p \leq \bar{p} .
$$

Then, the solution of (1) satisfying

$$
E(0)<E_{2}, \quad\left\|\nabla u_{0}\right\|_{2} \geq \alpha_{1},
$$

blows up in finite time. That is $\|u(t)\|_{p} \rightarrow \infty$ as $t \rightarrow T^{*}$ for some $0<T^{*}<\infty$.

Remark 5.2. The requirement $m>2$ in Theorem 5.1 is technical but it seems necessary in our proof. The case $m=2$ cannot be handled with the method we use here. But the same result can be shown for $m=2$ by using the concavity method. See [12] for more details.

Proof of Theorem 5.1. To prove Theorem 5.1, we suppose that the solution exists for all time and we reach to a contradiction. Following the idea introduced in [10] and developed in [20] and [27], we will define a function $\hat{L}$ which is a perturbation of the total energy of the system and which will satisfy the differential inequality

$$
\frac{d \hat{L}(t)}{d t} \geq \xi \hat{L}^{1+\nu}(t)
$$

where $\nu>0$. Inequality (66) leads to a blow up of the solution in finite time $T^{*} \geq \hat{L}(0)^{-\nu} \xi^{-1} \nu^{-1}$, provided that $\hat{L}(0)>0$. 
To do so, we define the functional $\hat{L}$ as follows:

$$
\hat{L}(t)=\mathscr{H}^{1-\sigma}(t)+\epsilon \int_{\Omega} u_{t} u d x+\epsilon \int_{\Gamma_{1}} u_{t} u d \Gamma
$$

where the functional $\mathscr{H}$ is defined in (36), $\sigma$ is satisfying

$$
0<\sigma \leq \min \left(\frac{p-m}{p(m-1)}, \frac{p-2}{2 p}, \frac{m-2}{2 m}, \hat{\sigma}\right)
$$

where $\hat{\sigma}$ is defined later in (71) and $\epsilon$ is a small positive constant to be chosen later. Taking the time derivative of $\hat{L}(t)$ and following the same steps as in the proof of Theorem 4.2, we get (instead of inequality (44)), for all $\lambda>0$,

$$
\begin{aligned}
l \hat{L}^{\prime}(t) \geq & l c_{m}(1-\sigma) \mathscr{H}^{-\sigma}(t)\left\|u_{t}\right\|_{m, \Gamma_{1}}^{m}-C_{m} \epsilon \frac{m-1}{m} \lambda^{-m /(m-1)}\left\|u_{t}\right\|_{m, \Gamma_{1}}^{m}+\epsilon l\left\|u_{t}\right\|_{2}^{2} \\
& +\epsilon l\|u\|_{p}^{p}+\epsilon l\left\|u_{t}\right\|_{2, \Gamma_{1}}^{2}-C_{m} \epsilon l \frac{\lambda^{m}}{m}\|u\|_{m, \Gamma_{1}}^{m} \\
& -\frac{\epsilon l}{2}(g \diamond u)(t)-\epsilon l\|\nabla u(t)\|_{2}^{2} .
\end{aligned}
$$

Next, for large positive $M$, we select $\lambda^{-m /(m-1)}=M \mathscr{H}^{-\sigma}(t)$. Then the estimate (69) takes the form:

$$
\begin{aligned}
l \hat{L}^{\prime}(t) \geq & \left(l c_{m}(1-\sigma)-M C_{m} \epsilon \frac{m-1}{m}\right) \mathscr{H}^{-\sigma}(t)\left\|u_{t}\right\|_{m, \Gamma_{1}}^{m}+\epsilon l\left\|u_{t}\right\|_{2}^{2} \\
& +\epsilon l\|u\|_{p}^{p}+\epsilon l\left\|u_{t}\right\|_{2, \Gamma_{1}}^{2}-C_{m} \epsilon l \frac{M^{-(m-1)}}{m} H^{\sigma(m-1)}\|u\|_{m, \Gamma_{1}}^{m} \\
& -\frac{\epsilon l}{2}(g \diamond u)(t)-\epsilon l\|\nabla u(t)\|_{2}^{2} .
\end{aligned}
$$

Exploiting (37) and (45), we get:

$$
\mathscr{H}^{\sigma(m-1)}\|u\|_{m, \Gamma_{1}}^{m} \leq C\|u\|_{p}^{(1-s) m+\sigma p(m-1)}\|\nabla u\|_{2}^{s m} .
$$

Thus, as in section 4, we have

$$
\|u\|_{p}^{(1-s) m+\sigma p(m-1)}\|\nabla u\|_{2}^{s m} \leq C\left[\left(\|u\|_{p}^{p}\right)^{\left(\frac{m(1-s)}{p}+\sigma(m-1)\right) \mu}+\left(\|\nabla u\|_{2}^{2}\right)^{\frac{m s \theta}{2}}\right] .
$$

Choosing $\mu, \theta$, and $s$ exactly as in section 4 (with strict inequalities), we choose $\sigma$ that verifies:

$$
\sigma \leq \frac{2-m s}{2(m-1)}\left(1-\frac{2 m(1-s)}{(2-m s) p}\right)=\hat{\sigma}
$$

The hypotheses on $m$ and $p$ ensure to have $0<\sigma<1$.

Consequently, we get from above:

$$
\mathscr{H}^{\sigma(m-1)}\|u\|_{m, \Gamma_{1}}^{m} \leq C\left[\left(\|u\|_{p}^{p}\right)^{\left(\frac{m(1-s)}{p}+\sigma(m-1)\right) \mu}+\|\nabla u\|_{2}^{2}\right] .
$$


Since,

$$
\left(\frac{m(1-s)}{p}+\sigma(m-1)\right) \frac{2}{2-m s} \leq 1
$$

applying the algebraic inequality (49), we get:

$$
\begin{aligned}
\left(\|u\|_{p}^{p}\right)^{\left(\frac{m(1-s)}{p}+\sigma(m-1)\right) \frac{2}{2-m s}} & \leq d\left(\|u\|_{p}^{p}+\mathscr{H}(0)\right) \\
& \leq d\left(\|u\|_{p}^{p}+\mathscr{H}(t)\right), \quad \forall t \geq 0 .
\end{aligned}
$$

Thus, (73) together with (72) leads to (see (53)):

$$
\begin{aligned}
\mathscr{H}^{\sigma(m-1)}\|u\|_{m, \Gamma_{1}}^{m} & \leq C d\left[\|u\|_{p}^{p}+l\|\nabla u\|_{2}^{2}+\mathscr{H}(t)\right] \\
& \leq C d\left[2 E_{2}+\left(1+\frac{2}{p}\right)\|u\|_{p}^{p}-\left\|u_{t}\right\|_{2}^{2}-\left\|u_{t}\right\|_{2, \Gamma_{1}}^{2}-(g \diamond u)(t)\right] .
\end{aligned}
$$

Inserting (74) into (70) and using (55), we obtain:

$$
\begin{aligned}
& l \hat{L}^{\prime}(t) \geq\left(l c_{m}(1-\sigma)-M C_{m} \epsilon \frac{m-1}{m}\right) \mathscr{H}^{-\sigma}(t)\left\|u_{t}\right\|_{m, \Gamma_{1}}^{m} \\
&+\epsilon \quad l\left(1+C_{m} \epsilon \frac{M^{-(m-1)}}{m} C d\right)\left\{\left\|u_{t}\right\|_{2}^{2}+\left\|u_{t}\right\|_{2, \Gamma_{1}}^{2}\right\}+2 \epsilon \mathscr{H}(t)-2 \epsilon E_{2} \\
&+\epsilon \quad\left\{l-\frac{2}{p}-C_{m} l \frac{M^{-(m-1)}}{m} C d\left(1+\frac{2}{p}\right)\right\}\|u\|_{p}^{p}+C_{m} \epsilon l \frac{M^{-(m-1)}}{m} C d(g \diamond u)(t) \\
&-2 \epsilon \quad C_{m} l \frac{M^{-(m-1)}}{m} C d E_{2}+\epsilon\left(1-\frac{l}{2}\right)(g \diamond u)(t) .
\end{aligned}
$$

Writing again $E_{2}=E_{2}\|u\|_{p}^{p} /\|u\|_{p}^{p}$ and using again (33), we deduce that:

$$
\begin{aligned}
l \hat{L}^{\prime}(t) & \geq\left(l c_{m}(1-\sigma)-M C_{m} \epsilon \frac{m-1}{m}\right) \mathscr{H}^{-\sigma}(t)\left\|u_{t}\right\|_{m, \Gamma_{1}}^{m} \\
& +\epsilon l\left(1+C_{m} \epsilon \frac{M^{-(m-1)}}{m} C d\right)\left\{\left\|u_{t}\right\|_{2}^{2}+\left\|u_{t}\right\|_{2, \Gamma_{1}}^{2}\right\}+2 \epsilon \mathscr{H}(t)+2 C_{m} \epsilon l \frac{M^{-(m-1)}}{m} C d E_{2} \\
& +\epsilon\left\{l-\frac{2}{p}-2 E_{2}\left(B_{1} \alpha_{2}\right)^{-p}-C_{m} l \frac{M^{-(m-1)}}{m} C d\left(1+\frac{2}{p}\right)-4 C_{m} l \frac{M^{-(m-1)}}{m} C d E_{2}\left(B_{1} \alpha_{2}\right)^{-p}\right\}\|u\|_{p}^{p} \\
& +\epsilon \quad C_{m} l \frac{M^{-(m-1)}}{m} C d(g \diamond u)(t) .
\end{aligned}
$$


Thus, using the definition of $c_{1}$ in Remark 4.5, we get:

$$
\begin{aligned}
l \hat{L}^{\prime}(t) & \geq\left(l c_{m}(1-\sigma)-M C_{m} \epsilon \frac{m-1}{m}\right) \mathscr{H}^{-\sigma}(t)\left\|u_{t}\right\|_{m, \Gamma_{1}}^{m} \\
& +\epsilon l\left(1+C_{m} \epsilon \frac{M^{-(m-1)}}{m} C d\right)\left\{\left\|u_{t}\right\|_{2}^{2}+\left\|u_{t}\right\|_{2, \Gamma_{1}}^{2}\right\}+2 \epsilon \mathscr{H}(t)+2 C_{m} \epsilon l \frac{M^{-(m-1)}}{m} C d E_{2} \\
& +\epsilon\left\{c_{1}-C_{m} l \frac{M^{-(m-1)}}{m} C d\left(1+\frac{2}{p}\right)-4 C_{m} l \frac{M^{-(m-1)}}{m} C d E_{2}\left(B_{1} \alpha_{2}\right)^{-p}\right\}\|u\|_{p}^{p} \\
& +\epsilon C_{m} l \frac{M^{-(m-1)}}{m} C d(g \diamond u)(t) .
\end{aligned}
$$

Since $c_{1}>0$, we choose $M$ large enough such that:

$$
c_{1}-C_{m} l \frac{M^{-(m-1)}}{m} C d\left(1+\frac{2}{p}\right)-4 C_{m} l \frac{M^{-(m-1)}}{m} C d E_{2}\left(B_{1} \alpha_{2}\right)^{-p}>0 .
$$

Once $M$ is fixed, we pick $\epsilon$ small enough such that

$$
l c_{m}(1-\sigma)-M C_{m} \epsilon \frac{m-1}{m}>0
$$

and $\hat{L}(0)>0$. This leads to

$$
\hat{L}^{\prime}(t) \geq \hat{\eta}\left(\left\|u_{t}\right\|_{2}^{2}+\left\|u_{t}\right\|_{2, \Gamma_{1}}^{2}+\mathscr{H}(t)+\|u\|_{p}^{p}+E_{2}\right)
$$

for some $\hat{\eta}>0$.

On the other hand, it is clear from the definition (67), we have:

$$
\hat{L}^{\frac{1}{1-\sigma}}(t) \leq C(\epsilon, \sigma)\left[\mathscr{H}(t)+\left(\int_{\Omega} u_{t} u d x\right)^{\frac{1}{1-\sigma}}+\left(\int_{\Gamma_{1}} u_{t} u d \Gamma\right)^{\frac{1}{1-\sigma}}\right] .
$$

By the Cauchy-Schwarz inequality and Hölder's inequality, we have:

$$
\begin{aligned}
\int_{\Omega} u_{t} u d x & \leq\left(\int_{\Omega} u_{t}^{2} d x\right)^{\frac{1}{2}}\left(\int_{\Omega} u^{2} d x\right)^{\frac{1}{2}} \\
& \leq C\left(\int_{\Omega} u_{t}^{2} d x\right)^{\frac{1}{2}}\left(\int_{\Omega}|u|^{p} d x\right)^{\frac{1}{p}},
\end{aligned}
$$

where $C$ is the positive constant which comes from the embedding $L^{p}(\Omega) \hookrightarrow L^{2}(\Omega)$. This inequality implies that there exists a positive constant $C_{1}>0$ such that:

$$
\left(\int_{\Omega} u_{t} u d x\right)^{\frac{1}{1-\sigma}} \leq C_{1}\left[\left(\int_{\Omega}|u|^{p} d x\right)^{\frac{1}{(1-\sigma) p}}\left(\int_{\Omega} u_{t}^{2} d x\right)^{\frac{1}{2(1-\sigma)}}\right] .
$$

Applying Young's inequality to the right hand-side of the preceding inequality, there exists a positive constant also denoted $C>0$ such that:

$$
\left(\int_{\Omega} u_{t} u d x\right)^{\frac{1}{1-\sigma}} \leq C\left[\left(\int_{\Omega}|u|^{p} d x\right)^{\frac{\tau}{(1-\sigma) p}}+\left(\int_{\Omega} u_{t}^{2} d x\right)^{\frac{\theta}{2(1-\sigma)}}\right]
$$


for $1 / \tau+1 / \theta=1$. We take $\theta=2(1-\sigma)$, hence $\tau=2(1-\sigma) /(1-2 \sigma)$, to get:

$$
\left(\int_{\Omega} u_{t} u d x\right)^{\frac{1}{1-\sigma}} \leq C\left[\left(\int_{\Omega}|u|^{p} d x\right)^{\frac{2}{(1-2 \sigma) p}}+\int_{\Omega} u_{t}^{2} d x\right] .
$$

Using the algebraic inequality (49) with $z=\|u\|_{p}^{p}, d=1+1 / \mathscr{H}(0), \omega=\mathscr{H}(0)$ and $\nu=\frac{2}{p(1-2 \sigma)}$ (the condition $(68)$ on $\sigma$ ensuring that $0<\nu \leq 1$ ) we get:

$$
z^{\nu} \leq d(z+\mathscr{H}(0)) \leq d(z+\mathscr{H}(t))
$$

Therefore, there exists a positive constant denoted $C_{2}$ such that for all $t \geq 0$,

$$
\left(\int_{\Omega} u_{t} u d x\right)^{\frac{1}{1-\sigma}} \leq C_{2}\left[\mathscr{H}(t)+\|u(t)\|_{p}^{p}+\left\|u_{t}(t)\right\|_{2}^{2}\right] .
$$

Following the same method as above, we can show that there exists $C_{3}>0$ such that:

$$
\left(\int_{\Gamma_{1}} u_{t} u d \Gamma\right)^{\frac{1}{1-\sigma}} \leq C_{3}\left[\mathscr{H}(t)+\|u(t)\|_{m, \Gamma_{1}}^{m}+\left\|u_{t}(t)\right\|_{2, \Gamma_{1}}^{2}\right] .
$$

Applying the inequality (51), we get:

$$
\left(\int_{\Gamma_{1}} u_{t} u d \Gamma\right)^{\frac{1}{1-\sigma}} \leq C_{4}\left[\mathscr{H}(t)+\|u(t)\|_{p}^{p}+l\|\nabla u(t)\|_{2}^{2}+\left\|u_{t}(t)\right\|_{2, \Gamma_{1}}^{2}\right] .
$$

Furthermore, inequality (60) leads to:

$$
\left(\int_{\Gamma_{1}} u_{t} u d \Gamma\right)^{\frac{1}{1-\sigma}} \leq C_{5}\left[\mathscr{H}(t)+\|u(t)\|_{p}^{p}+\left\|u_{t}(t)\right\|_{2, \Gamma_{1}}^{2}+E_{2}\right] .
$$

Collecting (77), (79) and (80), we obtain:

$$
\hat{L}^{\frac{1}{1-\sigma}}(t) \leq \hat{\eta}_{1}\left\{\left\|u_{t}(t)\right\|_{2}^{2}+\left\|u_{t}\right\|_{2, \Gamma_{1}}^{2}+\mathscr{H}(t)+\|u(t)\|_{p}^{p}+E_{2}\right\}, \quad \forall t \geq 0,
$$

for some $\hat{\eta}_{1}>0$.

Combining (76) and (81), then, there exists a positive constant $\xi>0$, as small as $\epsilon$, such that for all $t \geq 0$,

$$
\hat{L}^{\prime}(t) \geq \xi \hat{L}^{\frac{1}{1-\sigma}}(t)
$$

Thus, inequality (66) holds. Therefore, $\hat{L}(t)$ blows up in a finite time $T^{*}$.

On the other hand, from the definition of the function $\hat{L}(t)$ and using inequality (37), for small values of the parameter $\varepsilon$, it follows that:

$$
\hat{L}(t) \leq \kappa\left(\|u(t)\|_{p}^{p}\right)^{1-\sigma}
$$

where $\kappa$ is a positive constant. Consequently, from the inequality (83) we conclude that the norm $\|u(t)\|_{p}$ of the solution $u$, blows up in the finite time $T^{*}$, which implies the desired result. This completes the proof of Theorem 5.1. 


\section{References}

[1] R. A. Adams. Sobolev spaces. Academic Press, New York, 1975.

[2] K. T. Andrews, K. L. Kuttler, and M. Shillor. Second order evolution equations with dynamic boundary conditions. J. Math. Anal. Appl., 197(3):781-795, 1996.

[3] J. T. Beale. Spectral properties of an acoustic boundary condition. Indiana Univ. Math. J., 25(9):895-917, 1976.

[4] H. Brezis. Analyse fonctionnelle. Masson, Paris, 1983.

[5] B. M. Budak, A. A. Samarskii, and A. N. Tikhonov. A collection of problems on mathematical physics. Translated by A. R. M. Robson. The Macmillan Co., New York, 1964.

[6] R. W. Caroll and R. E. Showalter. Singular and Degenerate Cauchy Problems. Academic Press, New York, 1976.

[7] M. M. Cavalcanti, V. D. Cavalcanti, and I. Lasiecka. Well-posedness and optimal decay rates for the wave equation with nonlinear boundary damping-source interaction. J. Differential. Equations., 236:407-459, 2007.

[8] F. Conrad and O. Morgul. Stabilization of a flexible beam with a tip mass. SIAM J. Control Optim., 36(6):1962-1986, 1998.

[9] F. Gazzola and M. Squassina. Global solutions and finite time blow up for damped semilinear wave equations. Ann. I. H. Poincaré, 23:185-207, 2006.

[10] V. Georgiev and G. Todorova. Existence of a solution of the wave equation with nonlinear damping and source term. J. Differential. Equations., 109:295-308, 1994.

[11] S. Gerbi and B. Said-Houari. Local existence and exponential growth for a semilinear damped wave equation with dynamic boundary conditions. Advances in Differential Equations, 13(1112):1051-1074, 2008.

[12] S. Gerbi and B. Said-Houari. Asymptotic stability and blow up a semilinear damped wave equation with dynamic boundary conditions. Nonlinear Anal., (74):7137-7150, 2011.

[13] P. J. Graber and B. Said-Houari. Existence and asymptotic behavior of the wave equation with dynamic boundary conditions to appear in Appl. Math. Optim., http://dx.doi.org/10.1007/s00245-012-9165-1.

[14] M. Grobbelaar-Van Dalsen. On fractional powers of a closed pair of operators and a damped wave equation with dynamic boundary conditions. Appl. Anal., 53(1-2):41-54, 1994.

[15] M. Grobbelaar-Van Dalsen. On the initial-boundary-value problem for the extensible beam with attached load. Math. Methods Appl. Sci., 19(12):943-957, 1996.

[16] M. Kafini and S. A. Messaoudi. A blow-up result for a viscoelastic system in $\mathbb{R}^{n}$. Electron. J. Differential Equations, pages No. 113, 7 pp. (electronic), 2007. 
[17] M. Kafini and S. A. Messaoudi. A blow-up result in a Cauchy viscoelastic problem. Appl. Math. Lett., 21(6):549-553, 2008.

[18] J. L. Lions and E. Magenes. Problèmes aux limites non homogènes et applications. Vol. 1, 2. Dunod, Paris, 1968.

[19] W. Littman and L. Markus. Stabilization of a hybrid system of elasticity by feedback boundary damping. Ann. Mat. Pura Appl., IV. Ser. , 152:281-330, 1988.

[20] S. A. Messaoudi and B. Said-Houari. Global non-existence of solutions of a class of wave equations with non-linear damping and source terms. Math. Methods Appl. Sci., 27:16871696, 2004.

[21] S. A. Messaoudi and B. Said-Houari. Global nonexistence of positive initial-energy solutions of a system of nonlinear viscoelastic wave equations with damping and source terms. J. Math. Anal. Appl, 365(1):277-287, 2010.

[22] S. A. Messaoudi. Blow up in a nonlinearly damped wave equation. Mathematische Nachrichten., 231:1-7, 2001.

[23] S. A. Messaoudi. Blow up and global existence in nonlinear viscoelastic wave equations. Math. Nachrich, 260:58-66, 2003.

[24] T. Meurer and A. Kugi. Tracking control design for a wave equation with dynamic boundary conditions modeling a piezoelectric stack actuator. Int. J. Robust. Nonlinear Control, 21:542$562,2011$.

[25] G. Ruiz Goldstein. Derivation and physical interpretation of general boundary conditions. Adv. Differ. Equ., 11(4):457-480, 2006.

[26] H. Song and C. Zhong. Blow-up of solutions of a nonlinear viscoelastic wave equation. Nonlinear Anal. Real World Appl., 11(5):3877-3883, 2010.

[27] E. Vitillaro. Global existence theorems for a class of evolution equations with dissipation. Arch. Rational Mech. Anal., 149:155-182, 1999.

[28] S. Yu, M. Wang, and W. Liu. Blow up for a Cauchy viscoelastic problem with a nonlinear dissipation of cubic convolution type. Math. Methods Appl. Sci., 32(15):1919-1928, 2009.

[29] H. Zhang and Q. Hu. Energy decay for a nonlinear viscoelastic rod equations with dynamic boundary conditions. Math. Methods Appl. Sci., 30(3):249-256, 2007. 